Gennady V. Stepanov*, Dmitry Yu. Borin, Anton V. Bakhtiiarov and Pavel A. Storozhenko

\title{
Hybrid magnetic elastomers prepared on the basis of a SIEL-grade resin and their magnetic and rheological properties
}

\begin{abstract}
Hybrid magnetic elastomers (HMEs) belong to a novel type of magnetocontrollable elastic materials capable of demonstrating extensive variations of their parameters under the influence of magnetic fields. Like all cognate materials, HMEs are based on deformable polymer filled with a mixed or modified powder. The complex of properties possessed by the composite is a reflection of interactions occurring between the polymer matrix and the particles also participating in interactions among themselves. For example, introduction of magnetically hard components into the formula results in the origination of a number of significantly different behavioral features entirely unknown to magnetorheological composites of the classic type. Optical observation of samples based on magnetically hard filler gave the opportunity to establish that initial magnetization imparts magnetic moments to initially unmagnetized grains, as a result of which chain-like structures continue to be a feature of the material even after external field removal. In addition, applying a reverse field causes them to turn into the polymer as they rearrange into new ring-like structures. Exploration of the relationship between the rheological properties and magnetic field conducted on a rheometer using vibrational mechanical analysis showed an increase of the relative elastic modulus by more than two orders of magnitude or by $3.8 \mathrm{MPa}$, whereas the loss factor exhibited steady growth with the field up to a value of 0.7 being significantly higher than that demonstrated by elastomers with no magnetically hard particles. At the same time, measuring the electroconductivity of elastomers filled with a nickel-electroplated carbonyl iron powder made it possible to observe that such composites demonstrated an increase of variation of the resistivity of the composite influenced by magnetic field in comparison to elastomers containing untreated iron particles. The studies conducted indicate that this material exhibits both magnetorheological and
\end{abstract}

\footnotetext{
*Corresponding author: Gennady V. Stepanov, State Scientific Research Institute for Chemical Technologies of Organoelement Compounds, 105118 Moscow, Russia, E-mail: gstepanov@mail.ru. https://orcid.org/0000-0003-0053-1883

Dmitry Yu. Borin, Institute of Mechatronic Engineering, Technische Universität Dresden, 01062 Dresden, Germany. https://orcid.org/0000-0003-3842-1487
}

Anton V. Bakhtiiarov and Pavel A. Storozhenko, State Scientific Research Institute for Chemical Technologies of Organoelement Compounds, 105118 Moscow, Russia. https://orcid.org/0000-00022258-5850 (A.V. Bakhtiiarov)

Ә Open Access. @ 2020 Gennady V. Stepanov et al., published by De Gruyter. (c) BY-NC-ND This work is licensed under the Creative Commons Attribution-NonCommercial-NoDerivatives 4.0 International License.

https://doi.org/10.1515/psr-2020-0008 
magnetoresistive effect and does indeed have the potential for use in various types of devices.

Keywords: hybrid, magnetoactive, magnetoresistivity, magnetorheological, ring-like structures

\section{Introduction}

\subsection{Background}

This article is predominantly dedicated to a selective consideration of the magnetic and elastic features of hybrid magnetic composites. At the same time, in light of the fact that to a significant degree the "smart" status of the material is determined by the quality of the polymer to be flexible under stress of physical force, it is of certain interest to discuss its capability to conduct electric current depending on external magnetic field, also known as magnetoresistivity. Unlike conventional electroconductive substances, the family of composites being studied exhibits the capability of varying resistivity within a range of several orders of magnitude when influenced by moderate magnetic fields. Affected by a field, the particles tend to change their positions with respect to the neighbors to form structures more or less ordered into chains capable of conducting electric current. Owing to the fact that the intensity of this process depends on the possibility of every particle to make sufficient moves, this brings up the question of whether the polymer matrix can afford such deformations, which directly refers to its elastic properties.

As has become apparent from the experimental results obtained over the last year, these materials possess a significantly more extended set of specific features in comparison to classic magnetorheological elastomers (MREs). In view of the difficulties of studying the aforementioned properties in hybrid elastomers containing magnetically hard and soft components simultaneously, it seemed wise to investigate the magnetoresistivity phenomenon and magnetic/elastic features in pure form separately, which assumed preparation of samples solely filled with particles of either type.

A material based on magnetically hard filler is expected to exhibit good damping properties without the necessity to supply the overall unit with a bulky system of permanent magnets or electromagnets. Despite the fact that such a device may not be controlled externally, which will imminently result in a lower effectiveness, its simplicity will be a compensation leading to extensive application. As was expected initially, substances of this kind would possess a remnant magnetization and thus an internal magnetic field resulting in enhanced damping performance. In addition, it was established that under the influence of external magnetic fields, they exhibited an asymmetry of their elastic features determined by the direction of initial magnetizing [1-4]. All in all, these results found confirmation in Ref. [5]. 
Another distinctive quality of the new materials is that their loss-factor magnitudes were found to be noticeably higher in comparison to those of other MREs [6]. The new elastomer was assumed to show a strong magnetostriction, positive or negative depending on the directions of the initial magnetizing and external magnetic field vectors, which, however, had no experimental validation. As was established in later investigations, the phenomenon of rotation of magnetically hard particles embedded in the polymer as the external magnetic field changes direction was the cause. Owing to the fact that the samples were prepared on the basis of sufficiently soft silicone matrices, the elastic properties of the polymer made it possible for the rotation to occur in weak magnetic fields. This explains the fact that the samples practically always demonstrated codirectionality between the remanence vector and the external field independently of its direction. The interpretation of the rotation mechanism is brought in Refs. [7-10].

Studying the magnetorheological properties of hybrid magnetic elastomer (HME) on an oscillating rheometer in the plane-plane system has been found to be challenging. The difficulties are connected to the fact that the sample suffers significant deformation when subjected to magnetizing. The edges of a cylinder-shaped specimen wind strongly and roll up turning the cylinder into an ellipsoid. Work with such materials requires a special philosophy of measurement.

Soft matrices came useful in magnetoresistivity phenomenon studies. On the basis of the comparative method of investigation, the polymer was filled with untreated carbonyl iron particles and those subjected to electroplating with nickel. In light of the fact that nickel is more electroconductive and corrosion-proof than pure iron, samples with the nickel-electroplated magnetic filler were expected to show more extensive field-induced resistivity variations as compared to those containing no nickel. Measured at moderate magnetic fields using alternating current, the samples with nickel-plated particles did demonstrate the effect. However, as was found out later, the intensity of the phenomenon was not as strong as assumed initially. A possible reason for this is that galvanic treatment causes some agglomeration among the particles resulting in the formation of elongated grains. Embedded in polymer, such formations may experience difficulties moving, in light of which the elastic features of the matrix remain crucial.

\subsection{Elastomeric materials}

Initial attempts to prepare polymer-based magnetic composite materials were made using various elastic substances. Among them were natural rubber [11, 12] and aqueous gels prepared on the basis of polyvinyl alcohol and glutaraldehyde [13-16]. In his research, T. Mitsumata used $\mathrm{N}$-isopropylacrylamide and carrageenan [17-20]. Meanwhile, interest expressed toward natural and synthetic rubbers has been significantly 
more intense. In particular, M. Lokander has produced magnetorheological elastomers on the basis of natural and nitrile rubbers containing acrylonitrile and polybutadienerubber additives [21-23]. The most frequent use, at the same time, has been made of polyurethane and silicone resins. The advantages of two-can urethane compositions originate from the possibility to vary product elasticity in quite wide ranges and their higher durability in comparison to other elastomers having close elastic properties. Thus, 15 papers published by T. Mitsumata et al. have been dedicated to polyurethane elastomers as the basic material in research [14, 24]. Composites with polyurethane matrices have also been the object of study in the work conducted by A. Boczkowska [25-27].

Despite that, silicone elastomer remains a material most extensively employed in scientific investigations. In total, 70 to $80 \%$ of studies dedicated to magnetorheological and magnetoactive elastomers have been done using silicones. Like urethanes, their elastic properties may easily be preset in ranges even more extensive than those of the former. The second advantage is the simplicity of control of polymerization rate performed by temperature variation. Synthesis of a good sample of magnetic elastomer requires a degassing procedure following mixture preparation and followed by thermal treatment. This technology offers an easy possibility to obtain products with the desired mechanical features giving silicone resins advantage over urethanes. Indeed, in comparison to silicone, the rate of polymerization of urethane elastomer depends on the micro-amounts of catalyst introduced into the mixture being prepared. After that, there is only a limited time for the conduction of polymerization of the overall composition including inconvenient and time-consuming vacuuming, which requires certain knowledge and skills. At the same time, silicone is a commercial product and control of its elasticity may be performed even by a researcher representing an area other than polymer chemistry. Thus, according to the literature, polyaddition silicones are the polymers most frequently used by scientists. Unlike cold-cure compositions, their polymerization may quickly be initiated by simple heating. Their employment allows the researcher to spend as much time as necessary on the preparation of a sample with the desired formula and then quickly turn the semiproduct into polymer by heating. Bulky specimens may be easily prepared by cooking in the microwave oven [7]. Silicone elastomers of this type have been developed at Dow Corning (US Patent 3697473 (1973), US Patent 4322320, US Patent 4340709 (1983)) and Russian StateResearch Institute for Chemical Technologies of Organoelement Compounds (SIEL-grade resin, SU Patent 564315 (1975), SU Patent 639267 (1978), SU Patent 1086787 (1983)).

Most frequently, our samples have been fabricated on the basis of a SIEL-grade resin polymerizing according to the polyaddition mechanism; the semifabricate is a product of Russian State Research Institute for Chemical Technologies of Organoelement Compounds. 


\section{Synthesis and measurements}

\subsection{Synthesis of magnetic elastomers based on SIEL-grade silicone resin}

Owing to streamlined synthesis, a tailored elastomer is formed. The polyaddition reaction, whose path is shown schematically further, features one of the basic polymerization mechanisms (Figure 1.1):

As may be seen from the diagram, no secondary output is formed as a result of the polymerization, which allows to utilize such compositions not only as filmy or coating ones, but also as potting compounds to be used in enclosed volumes of every shape. Mold-cured silicone-based materials may have a range of physical states from gel-like or elastomeric to glass-like. The advantage of these elastomers is in the wide operation temperature interval extending from -90 to $+350{ }^{\circ} \mathrm{C}$. Depending on the task specifics, silicone-based polyaddition compounds may solidify at moderate temperatures from room temperature to $160{ }^{\circ} \mathrm{C}$ and over various time periods ranging from one day to $30 \mathrm{~min}$; these materials are capable of polymerizing with the formation of layers of different thicknesses. Owing to their good capability to cling to such surfaces as metal, glass, or ceramics, these elastomers demonstrate high adhesion to $\mathrm{NdFeB}$ or Fe particles coated with hydride-containing silicones.

The surface-modifying process is based on mixing a modifying agent and magnetic powders in toluene, during which the modifying agent precipitates on the surfaces of the grains forming a coating. Among other compounds, oligo-(methyl (or ethyl) hydrogen)siloxanes $[\mathrm{RSiHO}]_{n}\left(\mathrm{R}=\mathrm{CH}_{3}, \mathrm{C}_{2} \mathrm{H}_{5} ; n=10-15\right)$ are considered to be most effective. In a mixture with silicone oil, these silicones may be used for the creation of more bulky coatings intended to provide regularly spaced distribution of particles inside the silicone resin.

The hydrophobization mechanism of magnetic powders is based on the chemical interaction of their surface hydroxyl-groups, forming as a result of reacting with adsorbed water, with $\mathrm{Si}-\mathrm{H}$-bonds of the modifying agent leading to the addition of silicone groups to the surface of a particle and formation of water molecules (Figure 1.2):

According to this principle, all hydroxyl groups become substituted with siloxane groups, thus imparting wettability toward silicones to the surface of the magnetic particle.

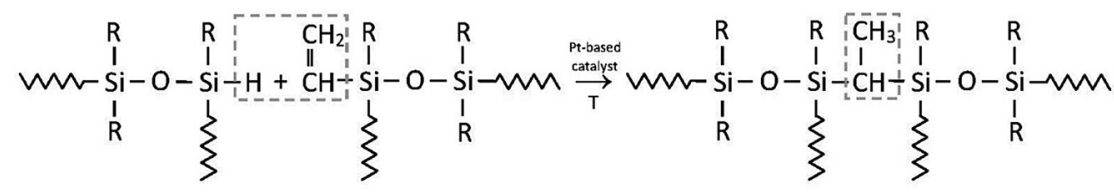

Figure 1.1: Polyaddition reaction; $\mathrm{R}$ being $\mathrm{CH}_{3}, \mathrm{C}_{2} \mathrm{H}_{5}, \mathrm{CH}_{2} \mathrm{CH}_{2} \mathrm{CF}_{3}$, or $\mathrm{C}_{6} \mathrm{H}_{5}$. 


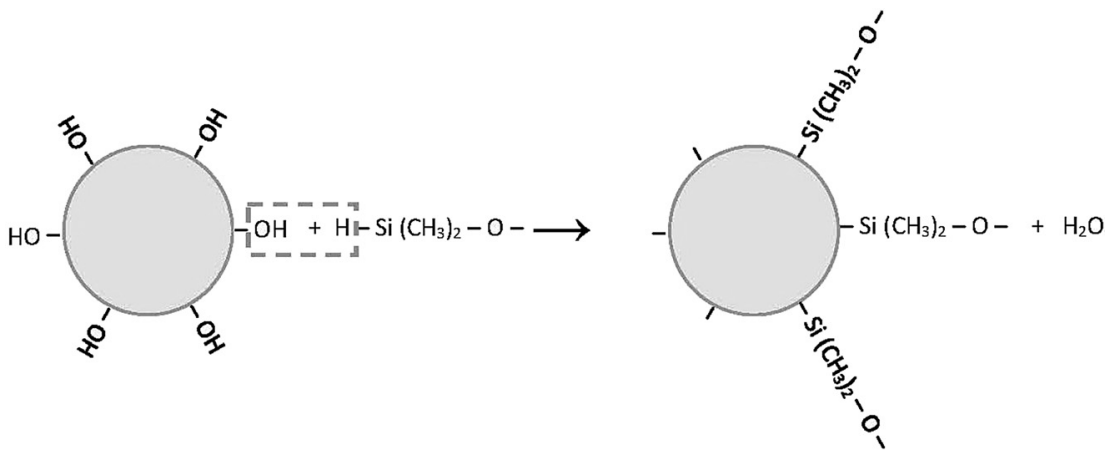

Figure 1.2: Interaction of hydroxyl groups with $\mathrm{Si}-\mathrm{H}$-bonds.

A study of thermal aging processes occurring in the polymers has revealed their heat stability retained over a long period of time, during which such basic parameters of elastomeric compositions as durability and relative strain continue to meet an adequate standard.

In view of the aforementioned properties, silicone elastomer is considered a promising material to be used as elastic matrix in magnetic elastomers fabrication.

\subsection{Magnetic properties of HME}

The key feature among the magnetic properties of a composite containing both magnetically hard and soft components is the newly discovered magnetization curve demonstrating an overall resemblance with but having a more complicated shape than that of a pure high-coercivity powder. Whereas an enhanced magnetic susceptibility peak, whose occurrence is determined by the presence of the magnetically soft component, most frequently being carbonyl iron, and another peak originating due to the availability of the magnetically hard component are observed in the areas of zerofield and filling-material demagnetizing force, respectively, increase of the magnetization loop amplitude or fabrication of samples based on softer matrices first results in the dying down of the "coercivity" peak followed by its shifting into the zero-field vicinity. At this, a composite filled with magnetically hard particles demonstrates the behavior rather characteristic of magnetically soft substances, owing to which most investigations directed at the detection and identification of processes taking place inside this magnetic elastomer were carried out on specimens prepared with no magnetically soft component. Therefore, all observations were limited to the phenomena demonstrated by magnetically hard particles.

In previous studies, attention was paid to the behavioral features of coercivity in HME, which took very low magnitudes [7-9] and might even be shifted into the area of 
fields of opposite polarity [10]. These effects are determined by the rotation of magnetically hard particles embedded in the polymer matrix. Magnetized, they start, at one moment, exhibiting the tendency to restore codirectionality with the external field after it changes its direction to opposite, rather that suffer interior polarity reversal themselves. A description of this effect is brought in our previous publications [7-10].

At the same time, the remnant magnetization demonstrated by the samples also tends to slide from high magnitudes, characteristic of magnetically hard filler, down to zero in intensity. It was supposed that whereas the cause of the phenomenon of zero magnetic flux on the surface of a magnetized specimen, which assumably had to demonstrate quite to the contrary, was not apparent initially, the explanation of this effect is based on the specifics of structuring of the magnetically hard filler inside the polymer. When the external magnetic field weakens to 0 , the magnetized particles show the tendency to form ring-like structures to confine the flux lines. Depending on the concentration of magnetic particles and their vicinities, the rings may contain various numbers of members starting from three (Figure 2.1). It should be noted that this assembling into circular formations is energetically favorable. The possibility of their existence had been considered for magnetically soft particles dispersed in liquid by S. Kantorovich in her theoretical work [28, 29] and by D. Borin and A. Zubarev in Ref. [30]. In a special experiment these assumptions were confirmed.

Typical magnetization curves recorded for two samples prepared on the basis of matrices with different rigidities (Figure 2.2) are presented further. Containing magnetically hard filler of the same type at equal concentrations of $25 \mathrm{vol} \%$, the specimens were obtained with shear moduli of 140 and $210 \mathrm{kPa}$, as was determined using a Thermo Scientific Haake MARS III rheometer. The essential behavioral dissimilarities they demonstrate are obvious: as may be noticed, whereas the rigid elastomer produces a wide hysteresis loop, that of the soft specimen is significantly narrower. In addition, the ascending curve pierces the zero-field area very close to the origin. It may, in particular, be seen from Figure 2.2 (b), which depicts the central section of the magnetization hysteresis loop recorded for a soft HME sample $\left(\mathrm{G}^{\prime}=140 \mathrm{kPa}\right)$ containing plate-like $\mathrm{NdFeB}$ particles.

Meanwhile, a more profound comprehension of the mechanisms of the processes occurring inside the composite under the influence of different magnetic fields may be
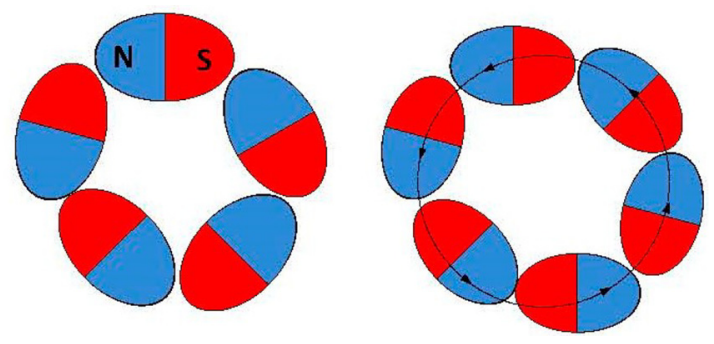

Figure 2.1: Ring-like structures formation phenomenon. 

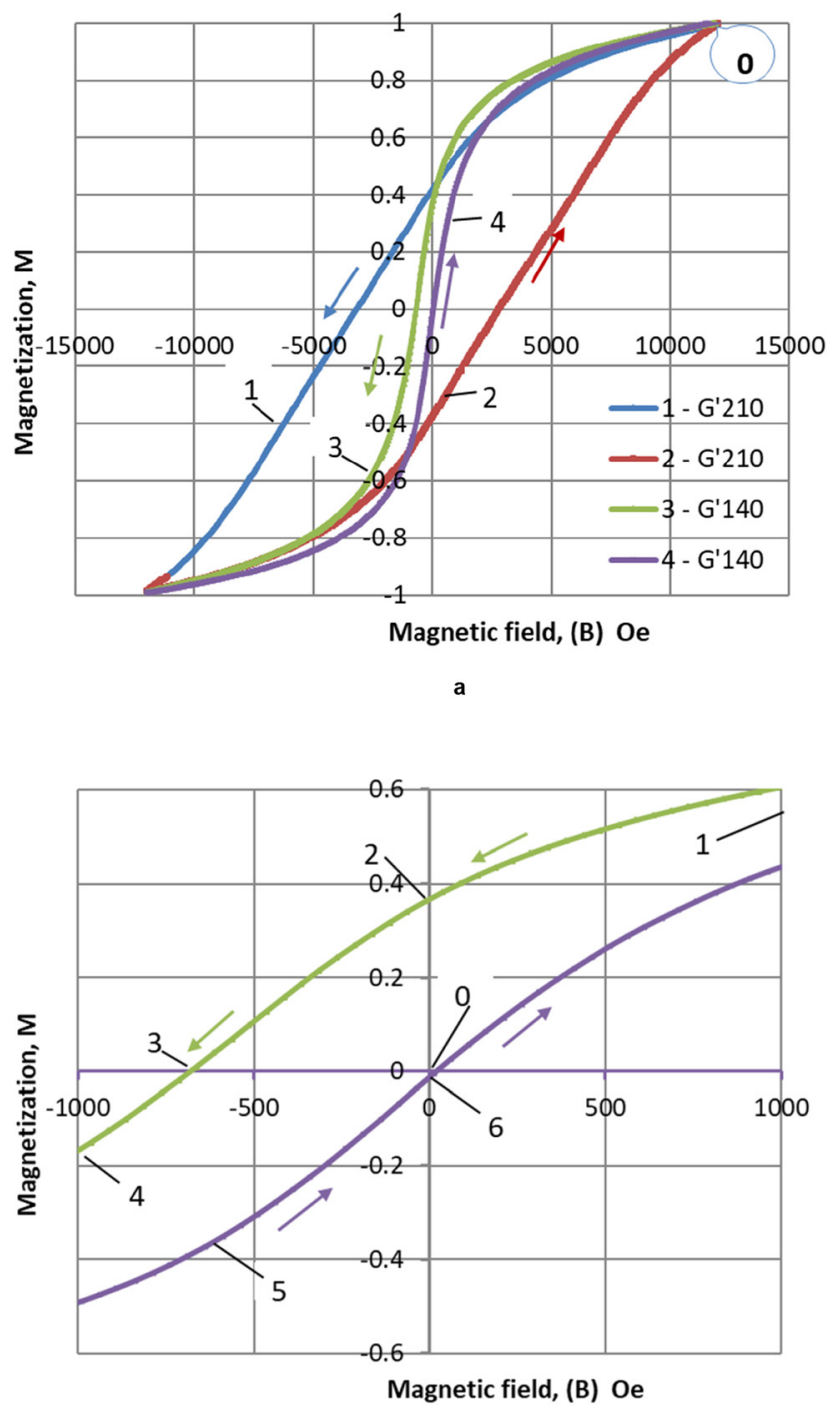

b

Figure 2.2: a) Magnetization hysteresis loops recorded for two magnetic elastomers filled with $\mathrm{NdFeB}$ particles and based on a rigid $\left(\mathrm{G}^{\prime}=210 \mathrm{kPa}\right.$, lines 1 and 2$)$ and soft $\left(\mathrm{G}^{\prime}=140 \mathrm{kPa}\right.$; lines 3 and 4) matrix; b) central section of the magnetization hysteresis loop corresponding to the most significant structural rearrangement occurring inside a soft HME sample. The path of magnetization variation with magnetic field is shown by the arrows. The initial magnetization state corresponds to point 0 . The numbered points correspond to the structures shown in Figure 2.3.

obtained by means of a qualitative experiment. Figure $2.3(\mathrm{a}-\mathrm{f})$ present photographic images of different arrangements of particles contained in a monolayer of the soft magnetic elastomer placed in different external conditions. 


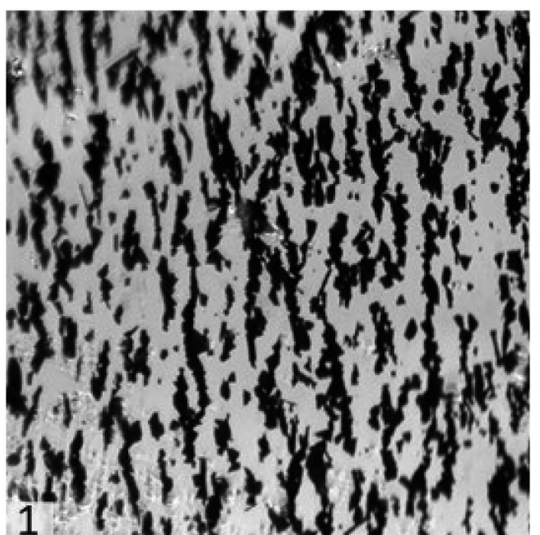

a
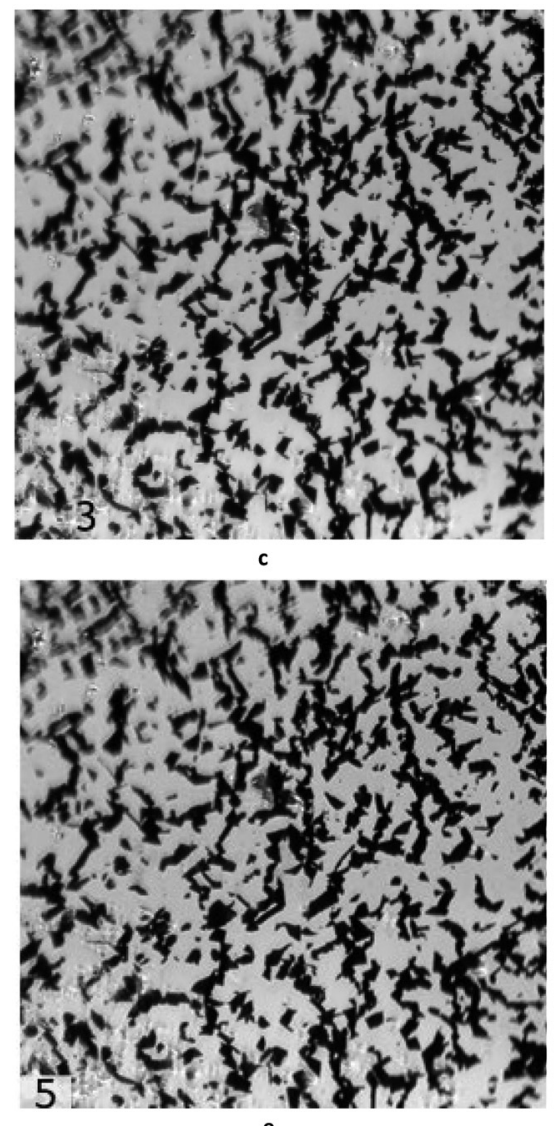

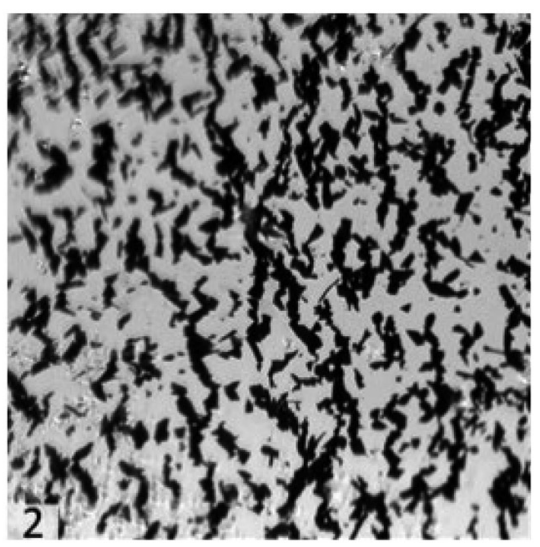

b

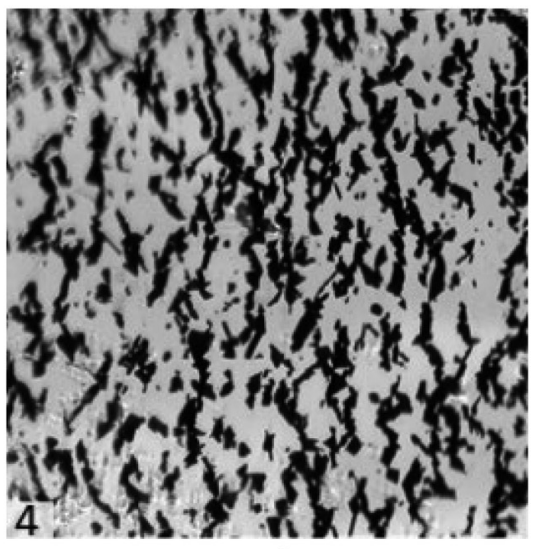

d

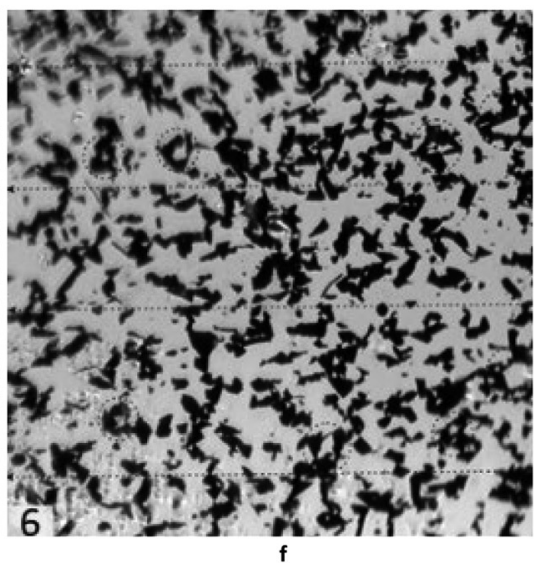

Figure 2.3: Structures formed by magnetized YMM-Q-grade NdFeB-alloy particles: a) in a magnetic field of 9000 Oe (point 1); b) at zero field reduced down to from 9000 Oe (point 2); c) in a reverse field of $800 \mathrm{Oe}$ (point 3); d) in a reverse field of $1550 \mathrm{Oe}$ (point 4); e) in a reverse field of $800 \mathrm{Oe}$ reduced down to from a reverse field of 1550 0e (point 5); f) at zero field reduced down to from a nonzero reverse field (point 6). Ring-like structures and the preferred orientation direction of the particles at zero field are denoted by dashed lines. 


\subsubsection{Plate-like particles}

The experiments, the results of which are presented further, were carried out with a monolayer specimen based on a soft matrix filled with plate-like YMM-Q-grade NdFeB-alloy particles with a coercivity of $250 \mathrm{mT}$. Before being studied in magnetic fields of different intensities and polarities, a nonmagnetic sample was subjected to magnetizing at 1.7 T, by which an internal structure was created. The images presented in Figure $2.3(\mathrm{a}-\mathrm{f})$ show the specifics of structural changes determined by the exterior influence complemented by the elastic forces of the polymer. The ordinal numbers of the images correspond to the numbered points on the magnetization curves presented in Figure 2.2.

Exhibiting a pronounced orientation in a field codirectional with initial magnetization (a), the chains partly lose ordering at zero field as a result of the tendency of the elastic forces of the polymer to destroy them (b). As the reverse magnetic field becomes stronger, the chains formed previously continue to break. Showing little change in weak reverse magnetic fields (c), the particles start turning over in growing field to restore codirectionality with its force lines becoming building material for a structure forming anew. Finally, exhaust of the potential for turning is observed, indicative of that all the particles are now engaged. On further increasing the reverse magnetic field, the chains demonstrate some elongation and straightening along the force lines (d). On the other hand, decreasing the reverse magnetic field causes destruction of the chains by the elastic forces of the polymer matrix (e). As the reverse field weakens to 0 , ringlike structures are formed, whereas the particles themselves and the previously built chains demonstrate a tendency to gain an orientation other than being colinear with the vector of the previously applied field (f). Thus, remnant magnetization is the factor preventing the destruction of the chains, which results in the formation of intermediate arrays. The polymer matrix remains strained inside owing to the deformations caused by the interacting magnetic particles it contains. The most illustrative video depicting structural rearrangements inside an initially magnetized sample being placed in a reverse magnetic field is available at Ref. [31].

\subsection{Measuring technique of the rheological properties of HME}

The rheological properties of NdFeB-based HME samples were studied on a Thermo Scientific HAAKE MARS III rheometer supplied with a magnetic measuring cell (Figure 3.1 (a)) consisting of a coil and a yoke. The cell is capable of generating magnetic fields ranging from 0 to $500 \mathrm{mT}$ and was calibrated by means of measuring the field inside it in the area where the specimen was supposed to be placed later, as a function of the electric current feeding the coil. It should be noted, however, that estimated at a level of $95 \%$ in the space immediately adjacent to the upper face of the stem of the yoke, the homogeneity of the magnetic field decreases with distance along the vertical axis dropping to $90 \%$ at $2 \mathrm{~mm}$ away from the surface. Therefore, whereas 
the greater heights of the specimen can provide a more pronounced rheological response, one always has to remember that such tests are limited by the necessity to strike a happy medium between the size of the sample and field uniformity.

As follows from Figure 3.1 (b), the specimen in the initial (I) state before being subjected to the magnetizing procedure bears against the rotor and base-plate surfaces

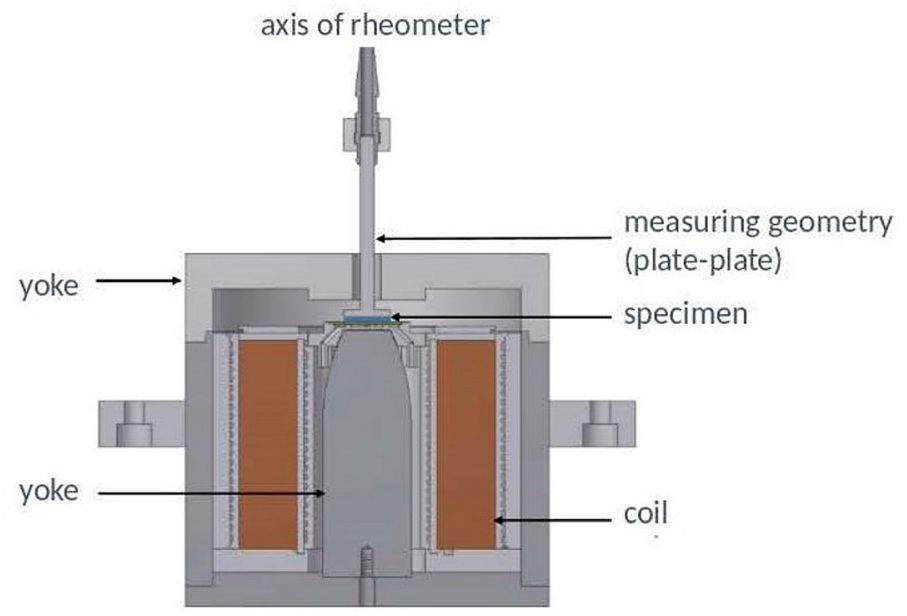

a

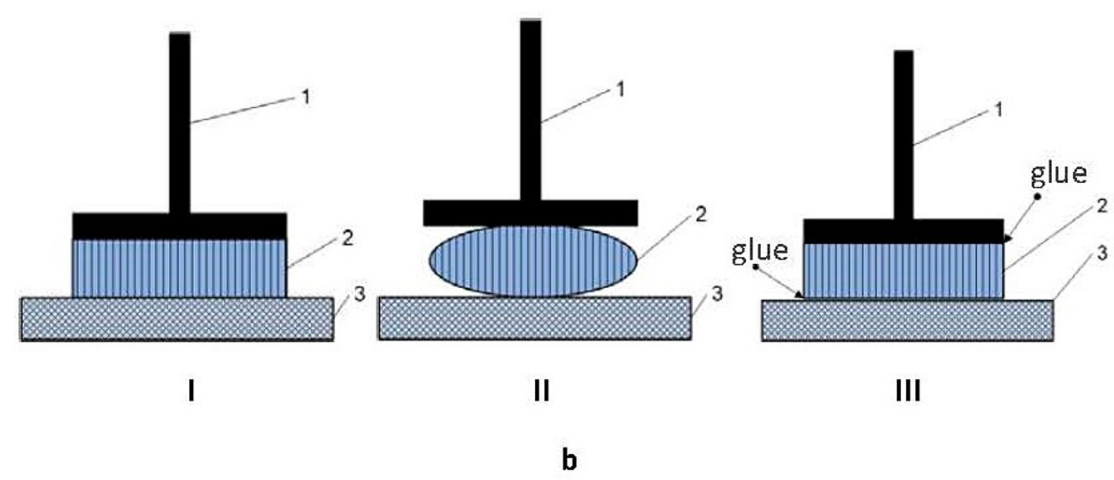

Figure 3.1: a) Magnetic measuring cell, a cross-sectional view (the device was designed by the Chair of Magnetofluiddynamics, Measuring and Automation Technology, TU Dresden ${ }^{1}$ ); b) HME sample in the rheometer: (I) before being magnetized; (II) in a magnetized state; and (III) glued to the surfaces of the rotor and base plate and magnetized. 1, 2, and 3 are the rotor, sample, and base, respectively.

1 The authors thank Prof. Stefan Odenbach for providing laboratory facilities of the Chair of Magnetofluiddynamics, Measuring and Automation Technology at TU Dresden. 
quite fully. However, after getting magnetized in the vertical direction (II), the elastomer shows a significant remanence, which results in strong attraction forces between the opposite poles of the sample causing its distortion into an ellipsoid. After that, even the application of strong compression forces along the vertical axis cannot restore the fullness of contact between the sample and the upper and lower surfaces. It is important to note that the standard technique of measurement using rheometers assumes sample compression by means of two planes from above and from below. At this, the rheological features of the material change significantly making all attempts to compare samples from different research groups meaningless, because of unacceptably high variations of compression from sample to sample and its relationship with the elasticity of a concrete specimen.

Gluing the sample to the surfaces of the rotor and base plate (III) makes it possible to minimize this irregularity and by that leave the sample undistorted, as a result of which measurement begins practically at zero compression. Caused by zero specimen sliding against the surfaces, the experimental indeterminacy is thus brought to a negligible level. In view of this, all the rheological measurements carried out within the frames of this project were performed on cylinder-shaped samples being 14 and $4 \mathrm{~mm}$ in diameter and height, respectively, and glued in according to the aforementioned method.

\subsection{Electric conductivity improvement by means of electroplating iron powders with nickel}

Electroplating of carbonyl iron particles was carried out according to the technology described in Ref. [32]. The procedure was conducted in a plastic container in an ethylene-glycol-based electrolyte composed by dissolving commercial nickel chloride in the solvent at concentrations ranging from 60 to $300 \mathrm{~g} / \mathrm{L}$. Despite the fact that galvanic operations are most frequently performed in aqueous solutions, selection of the glycol over water was determined by the capability of this solvent to wet even lyophobic surfaces. In addition, ethylene-glycol-based electrolytes allow high electric currents and remain efficient at temperatures close to boiling.

With the anode featuring a graphite bar immersed in the liquid and the cathode being a thin stainless-steel rod dipped into the bulk of powder, the process was started at ambient temperature. The powder being electroplated was held in contact with the cathode while simultaneously shuffled by a magnetic field created by a rotating constant magnet. During the process, as a result of the application of electric currents as strong as 1-2 A, the temperature of the electrolyte quickly increased to $60-80^{\circ} \mathrm{C}$. After such treatment for $1 \mathrm{~h}$ on average, the powders were decanted and washed with distilled water followed by rinsing with aqueous ammonia, then water again, and finally with isopropyl alcohol to be dried out at $60-70{ }^{\circ} \mathrm{C}$ in the oven.

Preliminary examination of the product recovered was performed by observation for the presence of hard agglomerates and impurities, after which their quality was 
evaluated by measuring their conducting properties. The measurements were done by placing small amounts of powder into a Teflon die between the face planes of two cylindrical-shaped rods being the upper and lower contacts, after which the readings of the ohmmeter $(\mathrm{R})$ were recorded. At this, the assemblage was loaded with a $1.5 \mathrm{~kg}$ weight installed on top of the upper contact, which, at a $5 \mathrm{~mm}$ inner diameter of the die, corresponded to a pressure of $7.5 \mathrm{kgf} / \mathrm{cm}^{2}$ applied axially. Aimed at being $3 \mathrm{~mm}$ in height, the amount of sample was controlled with a Vernier caliper. The necessity to know the geometrical parameters was dictated by the advantage of determining the more fundamental characteristics: the resistivities $(\rho)$ of the products were calculated according to the formula:

$$
\rho=\mathrm{R} \frac{\pi d^{2}}{4 l}
$$

with $\mathrm{R}, d$, and $l$ being the resistance of the sample, inner diameter of the die, and height of the sample, respectively. Samples of magnetic elastomer filled with electroplated powders were fabricated according to the technology described earlier. However, unlike samples produced for rheological tests, these specimens were polymerized in a polyurethane mold with a certain thickness $(h)$ and diameter $(D)$ of the cutout, pressed between two copper plates being the contacts.

Besides purely chemical investigations, the goals of this study assumed developing a better understanding of the relationship between the chemical formula of the magnetic filler and physical parameters of the overall composite. For this, the physicalmeasurement part of research was carried out on the basis of comparison of samples containing the products of electroplating with those solely filled with untreated carbonyl iron particles. All prepared isotropic, the specimens were subjected to the determination of their resistances (R) and capacitances (C) at different magnetic fields and various frequencies assigned by means of an E20-7 LCR-meter in the parallel-mode setting. The data recorded in the field and frequency ranges $0-420 \mathrm{mT}$ and $1-50 \mathrm{kHz}$, respectively, were processed into a series of frequency-reliant dependences of resistivity $(\rho)$ and permittivity $(\varepsilon)$ on external magnetic field (Eqs. (1) and (2), respectively). Ratios of resistivity and permittivity values determined at the strongest field and at zero magnetic field were used as measures of the effectiveness of the elastomers (Eqs. (3) and (4), respectively):

$$
\begin{gathered}
\varepsilon=\frac{4 C h}{\pi D^{2} \varepsilon_{0}} \\
\mathrm{n}_{\rho}=\frac{\rho_{\mathrm{B} \approx 0}}{\rho_{\min }} \\
\mathrm{n}_{\varepsilon}=\frac{\varepsilon_{\max }}{\varepsilon_{\mathrm{B} \approx 0}}
\end{gathered}
$$

with $D=18.0 \mathrm{~mm}$ and $h=1.5 \mathrm{~mm}, \mathrm{C}$ and $\varepsilon_{O}$ being the capacitance of the sample and electric permittivity of free space equal to $8.854 \cdot 10^{-12} \mathrm{~F} / \mathrm{m}$, respectively. 


\section{Discussion}

\subsection{Structuring mechanism}

As is evident from the observations presented earlier, the overall mechanism of structuring is determined by a few factors. On the one hand, external magnetic field, opposing the interior resistance of the material, tends to order magnetic particles along its vector. On the other hand, interactions occurring inside the composite feature a combination of two competing phenomena being the elastic resistance of the polymer and dipole interactions among magnetic particles. In addition, whereas the response of pure polymer is solely based on physical movements in the matrix and remains indifferent toward field influence, interplay among the particles exhibits a strong field dependence. At the same time, the vector of the strongest magnetic field ever applied to the material introduces the overriding factor. Indeed, a freshly prepared HME sample features unstrained polymerized resin filled with nonmagnetic powder and imposition of a magnetic field imparts magnetic moments to the particles. Interactions among the newly created magnetic dipoles immediately result in their multiple moves straining the polymer. Enhanced by the primary external field, magnetic interactions remain sufficiently intensive to hold the particles assembled into straight chains. Meanwhile, after turning the field off, magnetic forces pulling the particles together decrease in intensity, thus subjecting the chains to the influence of elastic forces tending to diminish interior tension and return the grains into their initial positions. However, as has been shown earlier, removal of the field is followed by only a partial destruction of the pattern. Owing to the strong remanence possessed by high-coercivity materials, magnetized particles continue to participate in dipole interactions remaining connected to each other. In addition, despite the reduction of their degree of order, the chains retain the direction, in which the primary structure was formed.

Abrupt switching to a magnetic field of opposite polarity creates a situation when its vector is contradirectional to the magnetic moments of the particles. In order to restore codirectionality, they might exhibit two different types of behavior depending on the elastic properties of the polymer: while tightly embedded in a rigid matrix, the grains demonstrate the classic mechanism of internal rearrangement, first changing their state to nonmagnetic, then to opposite polarity; soft matrices allow the particles to turn inside. Increasing the oppositely directed magnetic field does not lead to the formation of as well-ordered chains, however. Such an asymmetry may be explained by noticeably stronger elastic forces affecting the order of the internal chain-like structure being formed by turned-over particles as compared to when structuring requires no rotation and therefore doing additional work against the restoring forces of the polymer.

The most interesting case of structuring is observed when the reversal magnetic field is gradually reduced to 0 . As the field-induced magnetic forces holing the particles 
gathered in chains weaken, their tendency to turn back becomes more pronounced. As a result, at moderate fields, the chains are subjected to significant distortions making the impression that the system tends to rearrange the buildups in the way perpendicular to the influence vector. At the same time, magnetic interactions complemented by elastic forces are directed at diminishing the overall system energy. Driven by their remnant magnetization, particles assemble into rings confining circular magnetic field. It should be noticed that the ascending branch of the magnetization hysteresis loop lies close to the zero point, which corresponds to the low coercivity and remnant magnetization magnitudes related to it. In addition to that, it is possible to conclude that the asymmetry of the magnetization loop originates from the initial magnetizingfield memory phenomenon based on the existence of the easy magnetization direction possessed by every particle.

\subsection{Rheological properties of HME}

Determination of the rheological properties of HME was one of the purposes of the research. Meanwhile, owing to the complexity of the structuring mechanism, using the direct approach in their study was inefficient. As has been mentioned earlier, simplification of data interpretation suggested that all the experimental specimens be fabricated with magnetically hard particles only. At the same time, it was of interest to investigate the influence of particle size on the rheological behavior of the material. In order to do that, a commercial magnetic NdFeB-alloy powder with grains of sizes ranging up to $250 \mu \mathrm{m}$ was sieved into three fractions. Thus, three elastomer samples containing particles smaller than $40 \mu \mathrm{m}$, those falling in the range $40-80 \mu \mathrm{m}$, and grains bigger than $80 \mu \mathrm{m}$, were fabricated. The fourth sample filled with unfractionated polydisperse powder was synthesized for comparative purposes.

The relationship between shear modulus and deformation exhibited by the sample based on the smallest particles influenced by various magnetic fields is shown in Figure 3.2. As is possible to notice, the curves are of a standard shape and resemble those demonstrated by MRE containing magnetically soft particles. At deformations not exceeding $0.01 \%$, the shear modulus attains saturation and no longer exhibits the tendency to grow. Increasing deformation from 0.01 to $10 \%$ results in lower shear modulus magnitudes. At the same time, at stronger magnetic fields, the dependence of the shear modulus on deformation is pronounced more noticeably. Whereas at zero field, the shear modulus decreases by a factor of 3.3, which corresponds to a decrease from $25.4 \mathrm{kPa}$ observed at a $0.01 \%$ deformation to $7.5 \mathrm{kPa}$ observed at a $10 \%$ deformation, at $490 \mathrm{mT}$ the modulus changes by a factor of 35 decreasing from 3849 to $108 \mathrm{kPa}$. A similar relationship between the elasticity of filled elastomers and deformation, also known as the Payne effect, had been observed in samples filled with magnetically soft particles during testing on a rheometer in in-plane shear mode [33] and in stretch mode [34]. 
Comparison of the rheological behaviors demonstrated by the sample containing the finest fraction of particles and one filled with medium-size $(40-80 \mu \mathrm{m})$ particles makes it possible to see that the latter exhibits a similar relationship with deformation.

The lines lie very close to each other. On the one hand, it makes sense that the curve corresponding to the finest-filler sample runs slightly above that of its counterpart. On the other hand, the other sample might demonstrate a stronger elasticity increase when influenced by a magnetic field. However, the fact that this does not happen gives grounds for the conclusion that a high magnetorheological effect may indeed be observed with small particles only. At small deformations occurring in magnetic field, the elastic modulus increases by a factor of 150 or by in increment of $3.8 \mathrm{MPa}$, whereas at a $10 \%$ deformation the modulus increases by a factor of 15 , which corresponds to an increment of $120 \mathrm{kPa}$.

A comparative diagram for the loss factors demonstrated by the two samples as functions of deformation is brought in Figure 3.4.

Dependences of loss factor on deformation, grain size, and external magnetic field are tangly. At zero field, deformation increasing from 0.01 to $10 \%$ is followed by a small increase of the loss factor by 0.1. At the same time, magnetic field causes more significant incremental changes. It is interesting to note that its maximum value is observed at deformations ranging from 0.1 to $1 \%$. In addition, samples filled with bigger $(40-80 \mu \mathrm{m})$ particles show even higher loss-factor values.

The sample containing the biggest $(\geq 80 \mu \mathrm{m})$ particles shows results similar to those produced by the other two samples brought in Figure 3.2 and 3.3. However, the initial elastic modulus of this specimen equals $190 \mathrm{kPa}$, which is one order of magnitude greater in comparison with the other specimens. As a result, the sample demonstrates a

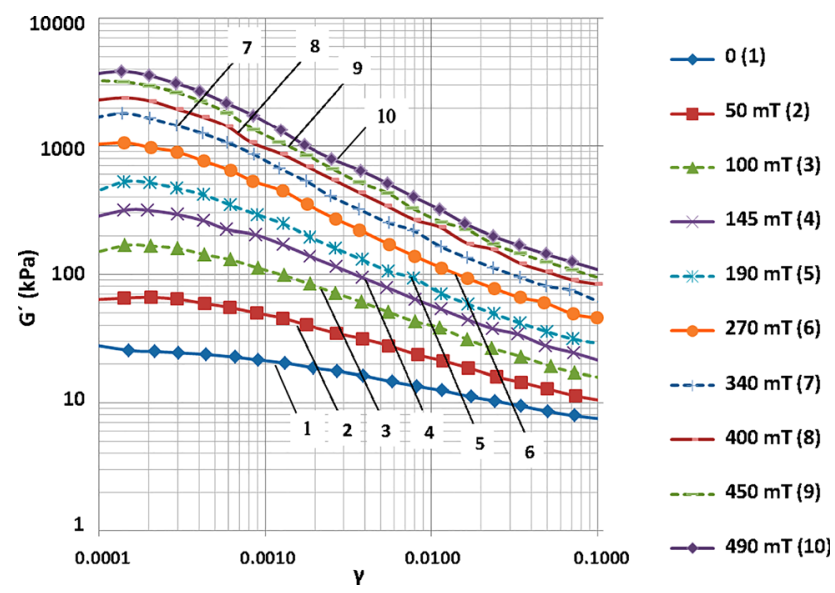

Figure 3.2: HME sample containing the smallest $(\leqslant 40 \mu \mathrm{m})$ particles. Shear modulus $\left(\mathrm{G}^{\prime}\right)$ as a function of deformation at different magnetic fields. 


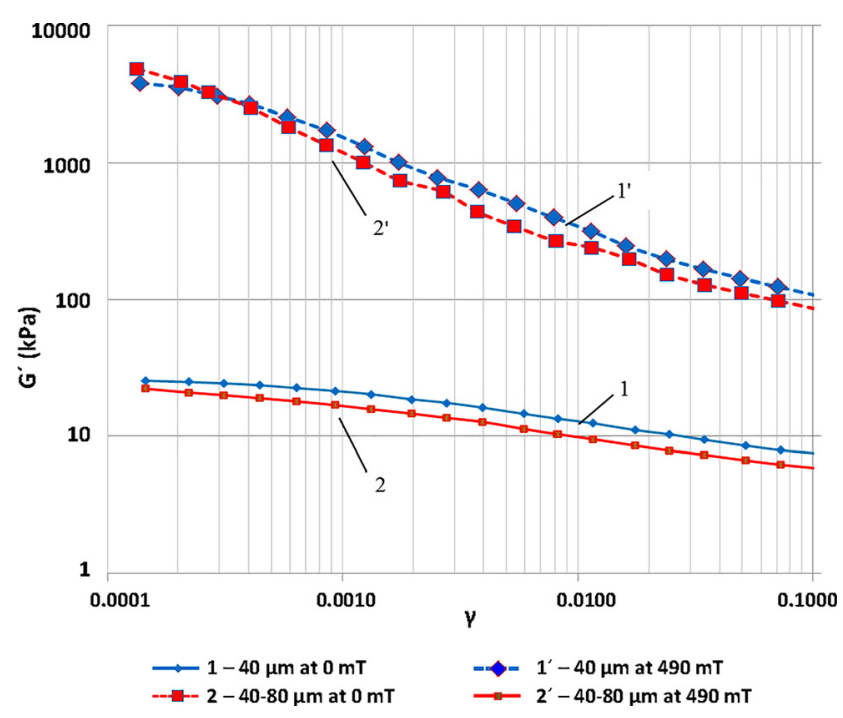

Figure 3.3: HME samples containing the smallest $(\leq 40 \mu \mathrm{m})$ and medium-size $(40-80 \mu \mathrm{m})$ particles. Comparison of their shear moduli $\left(\mathrm{G}^{\prime}\right)$ as functions of deformation at zero magnetic field and $490 \mathrm{mT}$.

very close absolute MR effect equal to 3.1 MPa, whereas at a deformation of $0.01 \%$ its relative magnitude turns out to be only 16 (Figure 3.5).

Finally, determination of the rheological parameters of the specimen containing unfractionated polydisperse particles was carried out for a better understanding of the tendencies demonstrated by the three samples considered previously and the dependences of their properties on filler dispersity. The proximity of its elastic modulus

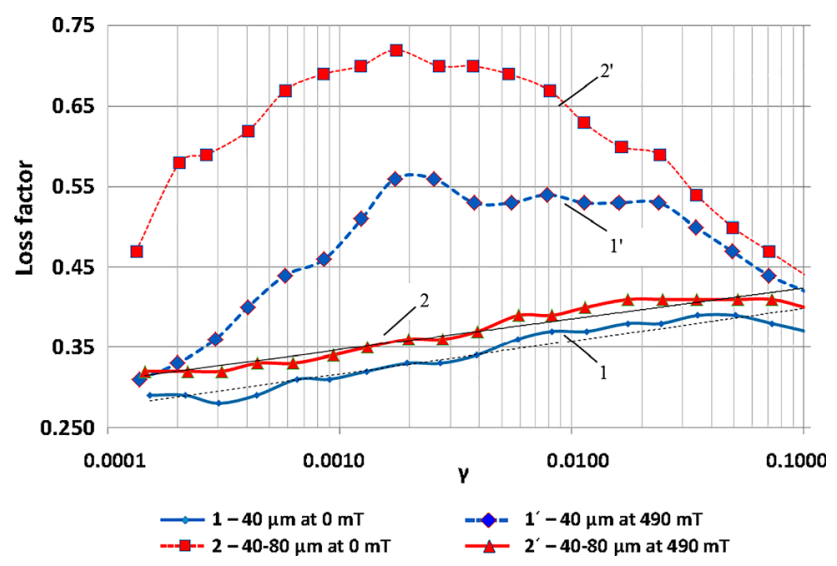

Figure 3.4: HME samples containing the smallest $(\leq 40 \mu \mathrm{m})$ and medium-size $(40-80 \mu \mathrm{m})$ particles. Comparison of their loss factors as functions of deformation at zero magnetic field and $490 \mathrm{mT}$. 


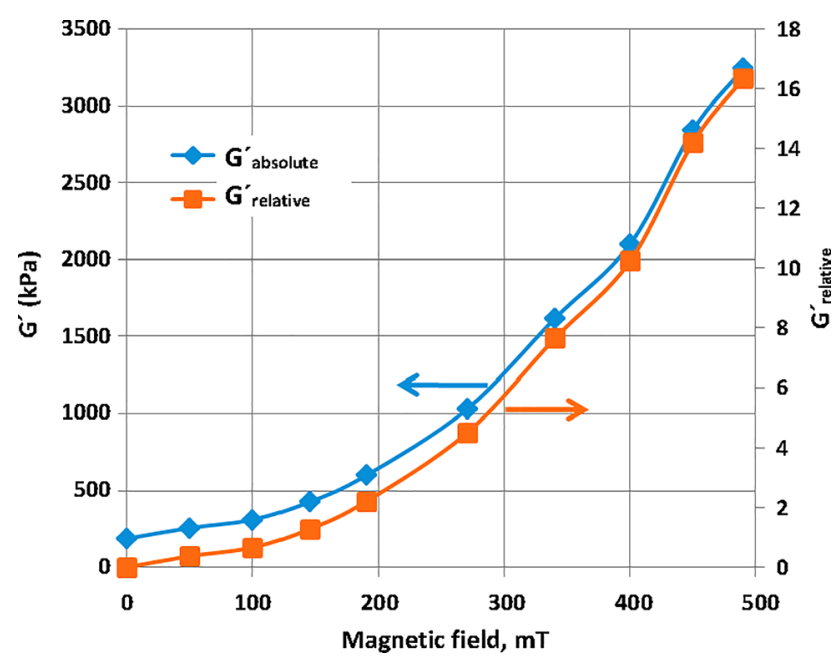

Figure 3.5: HME sample containing the largest $(\geq 80 \mu \mathrm{m})$ particles. Absolute and relative MR effect in relationship with external magnetic field.

being $28 \mathrm{kPa}$ to those of the three samples gives such a possibility. A comparative diagram for the sample filled with unfractionated particles and that containing the finest fraction is presented in Figure 3.6.

Meanwhile, comparison of the sample containing the smallest particles with those based on less-disperse powders unveiled very similar behavioral features in spite of the fact that the former shows a stronger magnetization and thus is expected to undergo a stronger structuring in magnetic fields. At the same time, the cumulative influence of such factors as high concentration of the filler, big size of its particles, and their platelike shapes may evidently be the cause of steric difficulties experienced by the grains attempting to line up along the magnetic field force lines.

It is necessary to note that whereas iron powders become saturated at $500 \mathrm{mT}$, $\mathrm{NdFeB}$-alloy particles require a 1-2 $\mathrm{T}$ field to attain saturation. As may be noticed from the following drawing, the magnetization and MR effect do not level out at $500 \mathrm{mT}$ and continue to grow, which is also seen well on the magnetization curves (Figure 3.7). A detailed description of these magnetic features is given in Ref. [16]. Most strongly the MR effect appears at small deformations. For instance, $G^{\prime}$ increases by a factor of 120 at a deformation of $0.01 \%$ and by a factor of 30 at a deformation of 1\% (Figure 3.8).

As may be seen from Figure 3.7, owing to the fact that the magnetization and remnant magnetization magnitudes demonstrate monotonous growth over the entire interval of fields ranging up to $1500 \mathrm{mT}$, the rheological studies carried out at fields not exceeding $500 \mathrm{mT}$ cannot unveil the complete potential of the composite material. 


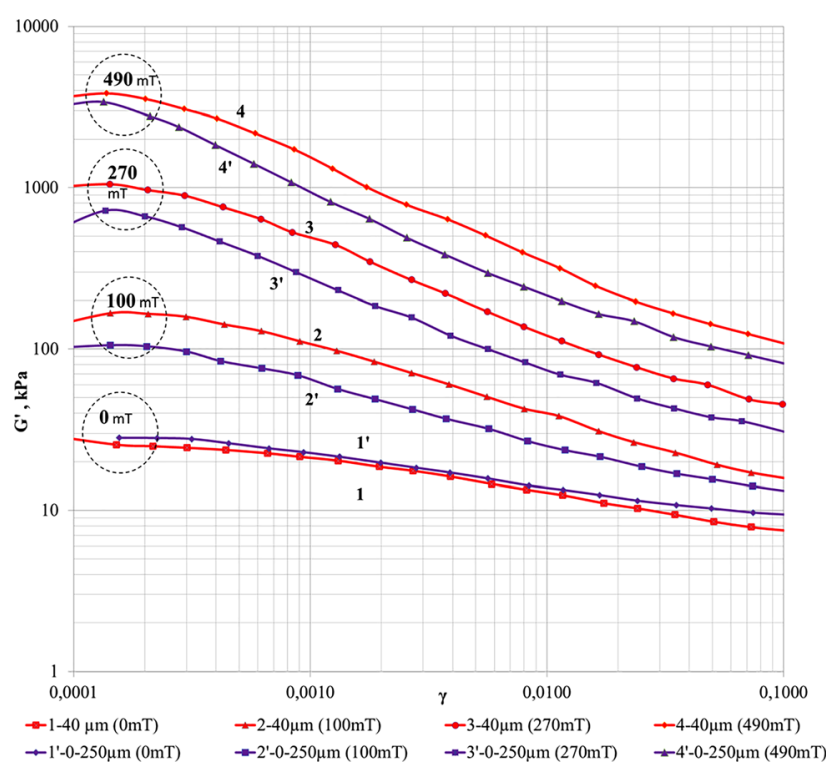

Figure 3.6: HME samples containing the smallest $(\leq 40 \mu \mathrm{m})$ particles and unfractionated filler $(0-250 \mu \mathrm{m})$. Comparison of their shear moduli $\left(\mathrm{G}^{\prime}\right)$ as functions of deformation at four magnitudes of external magnetic field.

The loss factor is an important index for MRE. Its magnitude is proportional to the capability of the material to dissipate energy and absorb vibrations. Comparison of specimens fabricated on the basis of particles with different sizes makes it possible to conclude that this parameter grows with external magnetic field strength and grain size.

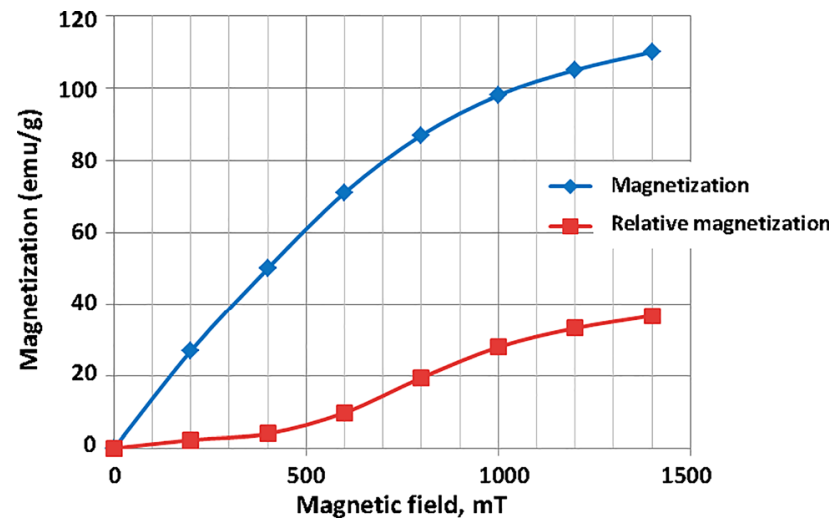

Figure 3.7: Magnetization and remnant magnetization as functions of external magnetic field. 


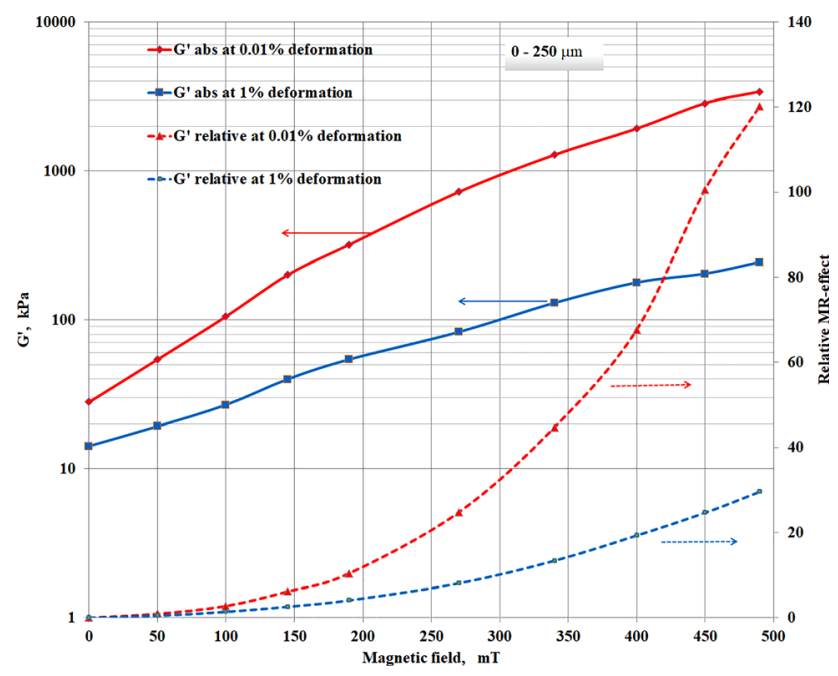

Figure 3.8: HME sample with polydisperse filler $(0-250 \mu \mathrm{m})$. Absolute and relative MR effect as a function of magnetic field at different deformations.

\subsection{Electrical properties of fillers and magnetic elastomers on their basis}

Accumulation of experimental results has offered a possibility to draw primary conclusions as well as do a step forward. On the one hand, carbonyl iron powders taken from different sources and having quite high resistivities become significantly more conductive after treatment. On the other hand, whereas ethylene-glycol-based electroplating has proven itself to be productive, we have not yet been able to make it fully controllable. All uncertainties evidently come from secondary chemical processes requiring additional study. So far, it has been noticed that the resistivities of the recovered products show a degree of variation; however, the set of factors having a direct effect of the merit of nickel-plated powders remain unknown. While nickel chloride concentration and high electric current most probably have a positive influence on the product quality, there is no clear understanding of such factors as the presence of $\mathrm{Fe}^{3+}$ cations in the electrolyte and its $\mathrm{pH}$ or freshly preparedness. Meanwhile, the range, within which most of the results have fallen, extends from 6.5 to $131.0 \mathrm{~m} \Omega \cdot \mathrm{m}$ being orders of magnitude narrower as compared to the spread of values demonstrated by the resistivities of untreated carbonyl iron powders. For example, whereas a commercial carbonyl iron powder containing spherical grains being $3-8 \mu \mathrm{m}$ in size exhibited a resistivity of $6.50 \cdot 10^{3} \Omega \cdot \mathrm{m}$, the product of its three-step grinding demonstrated a magnitude of $1.31 \Omega \cdot \mathrm{m}$. As is indicated by our experimental observations, the conductive properties of the initial material have a positive effect on the 
degree of conductivity improvement. In the example brought just earlier, this correlation may be explained by the fact that the grinding results in peeling an oxidized layer off the grains being treated. Thus, demonstrating a better conductivity before electroplating, such powders more stably and vigorously participate in the target electrochemical process. As has been established by means of the X-ray excited optical luminescence method, in most cases concentrations of metal nickel deposited on the iron particles fall within the limits of an interval extending from 1 to $4 \mathrm{wt}$.\%. Powders with better initial conductive properties correspond to higher nickel contents in the products, however.

The products of electroplating feature fluffy powders with color tones slightly darker than that of untreated carbonyl iron and contain no visible agglomerates. At the same time, although the overall method does prevent massive particle agglomeration manifesting itself as the formation of small stones, remnant magnetization turns up to be the factor responsible for the aggregation of particles into elongated gatherings (Figure 4.1).

As may be seen from the photographic images, the electroplated powder consists of agglomerates 50-100 $\mu \mathrm{m}$ long and 20-30 $\mu \mathrm{m}$ wide, which is noticeably more massive in comparison to the initial 3-8 $\mu \mathrm{m}$ spherical particles.

A powder containing $1 \mathrm{wt} . \%$ of metal nickel and exhibiting a resistivity of $45.5 \mathrm{~m} \Omega \cdot \mathrm{m}$ obtained from a carbonyl iron powder with an initial resistivity of $25-27 \Omega \cdot \mathrm{m}$ was used for the synthesis of experimental magnetic elastomer samples to be tested for magnetic field-sensing properties. Their physical parameters were measured against similar specimens fabricated on the basis of the initial carbonyl iron. All the samples were prepared isotropic and contained $80 \mathrm{wt} . \%$ of filler.

Presented in Figure 4.2, the graphs demonstrate frequency-reliant dependences of resistivity $(\rho)$ and permittivity $(\varepsilon)$ on external magnetic field. Whereas such features as the hysteresis phenomenon, the positive and negative frequency-dependent axial displacements of the $\rho$ - and $\varepsilon$-loops, respectively, and their geometrical orientations in the physical parameter - magnetic field coordinate systems remain similar, dissimilarities deserve a more attentive examination. First of all, the hysteresis loops recorded for the samples containing electroplated particles demonstrate higher widths resulting from a more reluctant response to the decreasing of external field. Not observed in samples filled with untreated iron powders, this retardation effect also manifests itself as a shifting of the extremum value from the corner of the loop along the reverse line to a lower magnetic field.

Prepared on the basis of soft polymer, the samples must allow easy particles displacements accompanied by their structuring in magnetic fields. Meanwhile, the reluctance observed in samples based on electrochemically treated powder containing large elongated particles most probably reflects the difficulties they experience moving inside the matrix resulting in an interval of fields, within which both the resistivity and permittivity continue to retain the variation tendency they showed while the external 

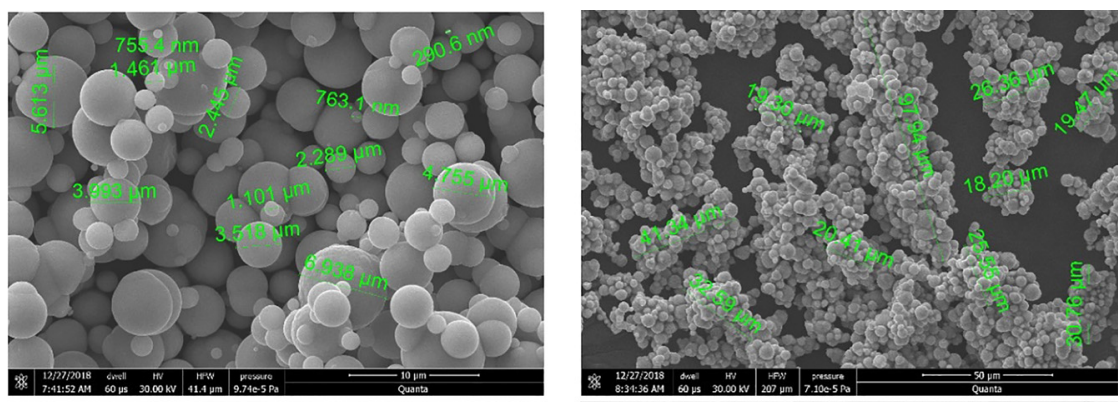

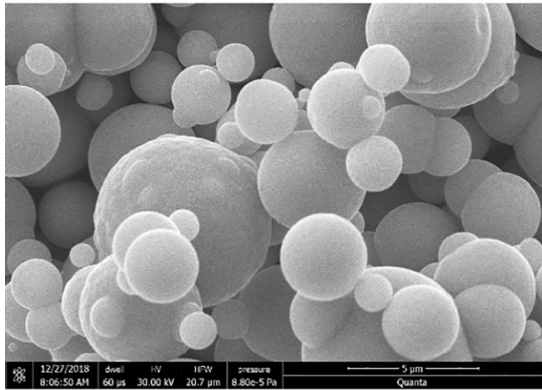

a

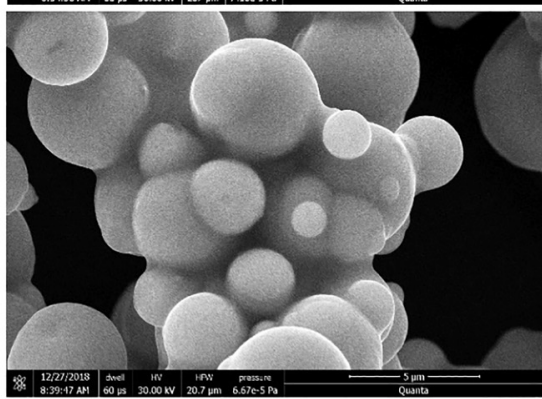

b

Figure 4.1: Microscopic images of (a) untreated carbonyl iron particles (scale bar $10 \mu \mathrm{m}$ above and $5 \mu \mathrm{m}$ below) and (b) nickel-electroplated carbonyl iron particles (scale bar $50 \mu \mathrm{m}$ above and $5 \mu \mathrm{m}$ below).

field was changing toward its maximum value. In the case of samples filled with spherical particles of untreated carbonyl iron, the corner of the hysteresis loop corresponding to the highest magnetic field is coincident with the extrema of the physical parameters being measured.

Substituting the extremal values and those the samples exhibit at zero field upon completion of a full hysteresis loop, into the expressions for $n_{\rho}$ and $n_{\varepsilon}$ brought in Part 2.4, we have established experimentally that introduction of nickelelectroplated particles indeed results in a higher performance of the magnetic elastomer. As may be seen from Tables 1 and 2 listing the magnitudes corresponding to the exceptional points of the frequency-reliant dependences and efficiency indices computed on their basis, the nickel-containing composite stably demonstrates a more extensive variation of its parameters, thus indicating a better magnetic field sensitivity.

It should be noted, however, that the capability to conduct electric current is determined not only by the low resistivity of the filler, but also by the possibility for its particles to line up in chains. Indeed, the magnetoresistivity phenomenon is based on internal structural changes similar to those described in Part 3.1. At the 

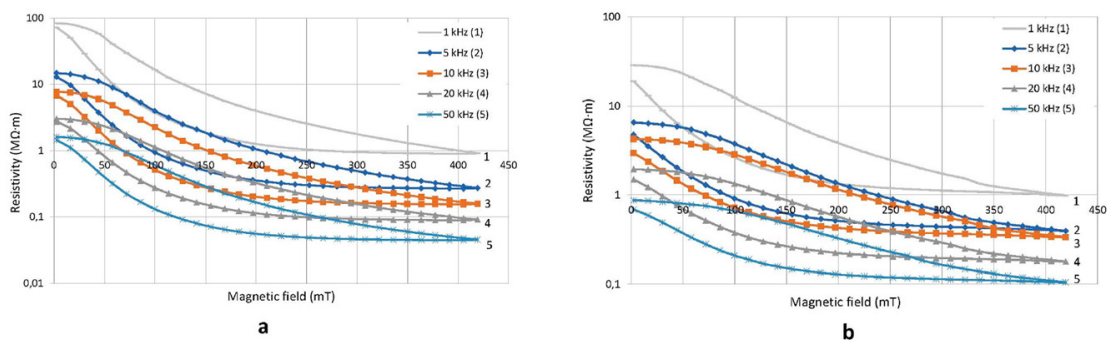

b

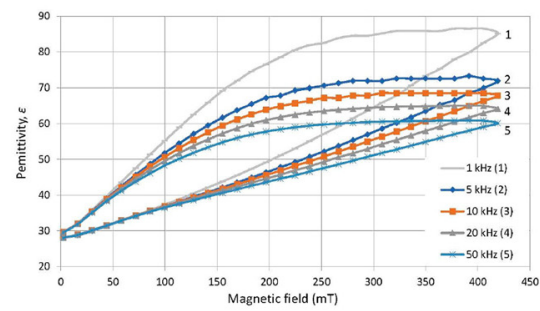

c

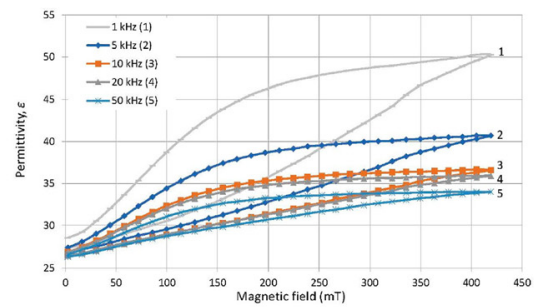

d
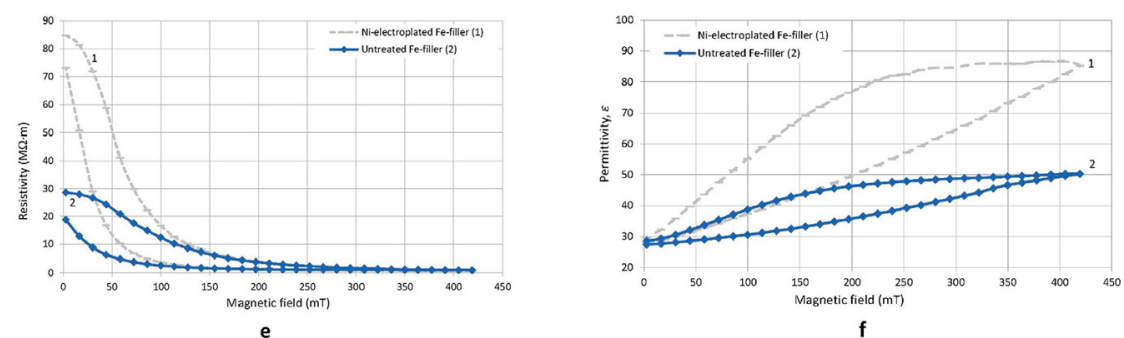

Figure 4.2: Physical parameters of samples of elastomer filled with magnetically soft particles as functions of external magnetic field: resistivity $(\rho)$ of elastomer filled with nickel-electroplated (a) and untreated (b) Fe particles; permittivity $(\varepsilon)$ of elastomer filled with nickel-electroplated (c) and Feuntreated (d) particles. Comparative diagrams showing the resistivity (e) and permittivity $(f)$ variations demonstrated by samples with different fillers under the influence of external magnetic field, measurements done at $1 \mathrm{kHz}$; the resistivity values are plotted along a linear scale.

same time, as is suggested by the closeness of the values of parameters observed at zero field in the initial $\left(B_{1} \approx 0\right)$ and final $\left(B_{2} \approx 0\right)$ points of the cycle, these materials containing no magnetically hard components are unlikely to develop an interior preferred orientation on primary magnetizing. Therefore, it may be assumed that the magnetic field sensitivity demonstrated by the sample filled with nickel-coated grains might probably be pronounced more strongly either with a more elastic matrix or if the electroplated particles had retained their spherical shapes, thus experiencing less intensive steric hindrances when driven by the magnetic field. 
Table 1: Resistivities at the starting point of the hysteresis loop $\left(B_{1} \approx 0\right)$, at the highest magnetic field $(B=420 \mathrm{mT})$, at a point of minimum $\left(\rho_{\mathrm{min}}\right)$, and upon completion of a full cycle $\left(B_{2} \approx 0\right)$ recorded for a sample filled with nickel-electroplated particles against one containing untreated carbonyl iron particles; resistivity-based performance index magnitudes determined for both specimens in combination with the relative resistivity-based performance characteristic of the former sample.

\begin{tabular}{|c|c|c|c|c|c|c|c|}
\hline $\begin{array}{l}\text { Frequency } \\
(\mathrm{kHz})\end{array}$ & Sample & $\begin{array}{r}\rho\left(B_{1} \approx 0\right) \\
(\Omega \cdot \mathrm{m})\end{array}$ & $\begin{array}{r}\rho(B=420 \mathrm{mT}) \\
(\Omega \cdot \mathrm{m})\end{array}$ & $\begin{array}{r}\rho_{\min } \\
(\Omega \cdot m)\end{array}$ & $\begin{array}{r}\rho\left(B_{2} \approx 0\right) \\
(\Omega \cdot m)\end{array}$ & $\mathrm{n}_{\rho}$ & $\begin{array}{r}n_{\rho} \\
(\mathrm{Fe} / \mathrm{Ni}) / n_{\rho}(\mathrm{Fe})\end{array}$ \\
\hline \multirow[t]{2}{*}{1} & $\mathrm{Ni} / \mathrm{Fe}$ & $8.48 \cdot 10^{7}$ & $9.21 \cdot 10^{5}$ & $8.99 \cdot 10^{5}$ & $7.31 \cdot 10^{7}$ & 81.3 & 4.26 \\
\hline & $\mathrm{Fe}$ & $2.86 \cdot 10^{7}$ & $9.85 \cdot 10^{5}$ & $9.85 \cdot 10^{5}$ & $1.88 \cdot 10^{7}$ & 19.1 & \\
\hline \multirow[t]{2}{*}{5} & $\mathrm{Ni} / \mathrm{Fe}$ & $1.48 \cdot 10^{7}$ & $2.73 \cdot 10^{5}$ & $2.68 \cdot 10^{5}$ & $1.30 \cdot 10^{7}$ & 48.5 & 4.01 \\
\hline & $\mathrm{Fe}$ & $6.53 \cdot 10^{6}$ & $3.94 \cdot 10^{5}$ & $3.94 \cdot 10^{5}$ & $4.76 \cdot 10^{6}$ & 12.1 & \\
\hline \multirow[t]{2}{*}{10} & $\mathrm{Ni} / \mathrm{Fe}$ & $7.75 \cdot 10^{6}$ & $1.58 \cdot 10^{5}$ & $1.55 \cdot 10^{5}$ & $6.85 \cdot 10^{6}$ & 44.2 & 4.97 \\
\hline & $\mathrm{Fe}$ & $4.27 \cdot 10^{6}$ & $3.34 \cdot 10^{5}$ & $3.34 \cdot 10^{5}$ & $2.96 \cdot 10^{6}$ & 8.9 & \\
\hline \multirow[t]{2}{*}{20} & $\mathrm{Ni} / \mathrm{Fe}$ & $3.07 \cdot 10^{6}$ & $9.13 \cdot 10^{4}$ & $8.89 \cdot 10^{4}$ & $2.78 \cdot 10^{6}$ & 31.3 & 3.77 \\
\hline & $\mathrm{Fe}$ & $1.95 \cdot 10^{6}$ & $1.79 \cdot 10^{5}$ & $1.79 \cdot 10^{5}$ & $1.49 \cdot 10^{6}$ & 8.3 & \\
\hline \multirow[t]{2}{*}{50} & $\mathrm{Ni} / \mathrm{Fe}$ & $1.62 \cdot 10^{6}$ & $4.55 \cdot 10^{4}$ & $4.44 \cdot 10^{4}$ & $1.46 \cdot 10^{6}$ & 32.9 & 5.06 \\
\hline & $\mathrm{Fe}$ & $8.70 \cdot 105$ & $1.05 \cdot 105$ & $1.05 \cdot 105$ & $6.86 \cdot 105$ & 6.5 & \\
\hline
\end{tabular}

Table 2: Permittivities at the starting point of the hysteresis loop $\left(B_{1} \approx 0\right)$, at the highest magnetic field ( $B=420 \mathrm{mT})$, at a point of maximum $\left(\varepsilon_{\max }\right)$, and upon completion of a full cycle $\left(B_{2} \approx 0\right)$ recorded for a sample filled with nickel-electroplated particles against one containing untreated carbonyl iron particles; permittivity-based index performance magnitudes determined for both specimens in combination with the relative permittivity-based performance characteristic of the former sample.

\begin{tabular}{llrrrrrr}
\hline $\begin{array}{l}\text { Frequency } \\
(\mathbf{k H z})\end{array}$ & Sample & $\boldsymbol{\varepsilon}\left(\boldsymbol{B}_{\mathbf{1}} \approx \mathbf{0}\right)$ & $\boldsymbol{\varepsilon}(\boldsymbol{B}=420 \mathrm{mT})$ & $\boldsymbol{\varepsilon}_{\max }$ & $\boldsymbol{\varepsilon}\left(\boldsymbol{B}_{2} \approx 0\right)$ & $\boldsymbol{n}_{\boldsymbol{\varepsilon}}$ & $\begin{array}{r}\boldsymbol{n}_{\boldsymbol{\varepsilon}} \\
(\mathrm{Fe} / \mathrm{Ni}) / \boldsymbol{n}_{\boldsymbol{\varepsilon}}(\mathrm{Fe})\end{array}$ \\
\hline 1 & $\mathrm{Ni} / \mathrm{Fe}$ & 28.0 & 85.2 & 86.5 & 29.7 & 2.91 & 1.65 \\
& $\mathrm{Fe}$ & 27.4 & 50.3 & 50.3 & 28.6 & 1.76 & \\
5 & $\mathrm{Ni} / \mathrm{Fe}$ & 28.0 & 71.9 & 73.2 & 29.6 & 2.47 & 1.66 \\
& $\mathrm{Fe}$ & 26.8 & 40.7 & 40.7 & 27.4 & 1.49 & \\
10 & $\mathrm{Ni} / \mathrm{Fe}$ & 28.1 & 67.9 & 68.6 & 29.6 & 2.32 & 1.69 \\
& $\mathrm{Fe}$ & 26.4 & 36.7 & 36.7 & 26.8 & 1.37 & \\
20 & $\mathrm{Ni} / \mathrm{Fe}$ & 28.1 & 64.2 & 65.0 & 29.6 & 2.20 & 1.64 \\
& $\mathrm{Fe}$ & 26.4 & 35.9 & 35.9 & 26.8 & 1.34 & \\
50 & $\mathrm{Ni} / \mathrm{Fe}$ & 28.0 & 60.1 & 60.9 & 29.5 & 2.06 & 1.61 \\
& $\mathrm{Fe}$ & 26.3 & 34.0 & 34.0 & 26.6 & 1.28 & \\
\hline
\end{tabular}

\section{Conclusions}

Study of the behavior exhibited by composite materials containing magnetic filler is essentially based on research on systems consisting of strongly interacting elements. Their mutual influence is complex and results in the origination of effects having a 
certain subordination manifesting itself as an asymmetry of physical properties and memory effects.

Analysis of the experimental observations has led us to the understanding that introduction of high-coercivity components into the formula is determinative of the mechanism of interactions among magnetized particles contained in the elastomer. The detailed consideration of the formation of various patterns brought in Parts 2.2 and 3.1 helps develop a vision of how the interior processes affect the behavior of HME observed during testing. In particular, such phenomena as low coercivity and weak remnant magnetization exhibited by the sample accompanied by its overall polarity reversal at moderate reverse magnetic fields take place owing to the capability of the magnetized particles embedded in its soft matrix to turn. At this, conditioned by a certain relation among the demagnetizing force of the pure filler, its remanence, and polymer elasticity, the rotation effect may turn out to be quite intensive resulting in a significant asymmetry of the magnetization hysteresis loop showing as shifting its ascending branch to the left of the origin and thus demonstrating abnormal signs of coercivity and magnetization at moderate reverse magnetic fields [10]. In addition, the closeness of the magnetic moment demonstrated by HME on the ascending magnetization curve is reflective of rearrangements among the magnetized particles leading to the formation of ring-like assemblages as the reverse magnetic field is decreasing in intensity approaching zero. At the same time, the specifics of the structuring processes remain geometrically predetermined by the direction of initial magnetization manifesting itself as a disproportional response to external magnetic vector variations.

Determined by these interior structuring processes, the rheological properties exhibited by HME also have certain specifics. For instance, unlike "classic" elastomers filled with magnetically soft particles, samples of hybrid magnetic composite demonstrate a tendency of the loss factor to increase whereas the field, the material is exposed to, becomes stronger. Showing an elastic modulus several times that of MRE with magnetically soft filler, HME, nevertheless, demonstrates a similar tendency of its viscosity and elastic modulus to grow with field.

This resemblance of the material of interest with elastomers containing magnetically soft particles gives a chance to consider the influence, the shapes of particles have on its overall performance, from a different angle. Owing to the fact that measurements of the magnetoresistivity effect assuming successive testings of the same sample over the same interval of magnetic fields are impossible, the sameness of field-induced stiffening comes useful making it possible to substitute a sample containing highcoercivity particles with one filled with magnetically soft powder. As has been mentioned in Part 3.3, electroplating results in some agglomeration leading to the formation of elongated assemblages. Although the nickel-coated particles occur orders of magnitude more electroconductive in comparison to their untreated carbonyl iron counterparts, alone, this property remains a useful but insufficient factor for making the overall composite better conducting. At the same time, the experimental results suggest that the softness of the polymer matrix and the capability of the particles of 
forming chain-like structures under the influence of magnetic fields are the required supplementary conditions. Whereas the performance of a sample filled with electroplated particles, which have retained their initial spherical morphologies, has not yet been observed, using experimentally obtained powders has indeed boosted magnetic field sensitivity. The resistivity- and permittivity-based performance indices demonstrated by a sample prepared with nickel-coated particles exceed those shown by one containing untreated carbonyl iron by a factor of 3.77-5.06 and 1.61-1.69, respectively. Meanwhile, the wider hysteresis loops resulting from a more sterically hindered, and thus reluctant, motion of the electroplated particles are probably indicative of a somewhat more pronounced tendency of the material to retain interior structures.

Keeping in mind that this research has been inspired by the purpose to develop a novel material with extraordinary features, collection of scientific facts about elastomeric materials containing magnetically hard particles is definitely a step forward. Study of the magnetoresistivity phenomenon is a contribution to the knowledge about sensors.

Author contribution: All the authors have accepted responsibility for the entire content of this submitted manuscript and approved submission.

Research funding: The reported study was funded by RFBR according to the research project 19-53-12039 and by Deutsche Forschungsgemeinschaft (DFG) under Grant BO 3343/2-1 within PAK 907.

Conflict of interest statement: The authors declare no conflicts of interest regarding this article.

\section{References}

1. Stepanov GV, Chertovich AV, Kramarenko EY. Magnetorheological and deformation properties of magnetically controlled elastomers with hard magnetic filler. J Magn Magn Mater 2012;324: 3448-51.

2. Linke JM, Borin DY, Odenbach S. First-order reversal curve analysis of magnetoactive elastomers. RSC Adv 2016;6:100407-16.

3. Borin DY, Stepanov GV, Odenbach S. Tuning of the tensile modulus of the magnetorheological elastomer with magnetic hard powder. J Phys Conf 2013;412:012040.

4. Borin DY, Stepanov GV. Oscillation measurements on magnetoactive elastomers with complex composition. J Optoelectron Adv Mater 2013;15:249-53.

5. Kramarenko EY, Chertovich AV, Stepanov GV, Semisalova AS, Makarova LA, Perov NS, et al. Magnetic and viscoelastic response of elastomers with hard magnetic filler. Smart Mater Struct 2015;24:035002.

6. Wen $\mathrm{Q}$, Wang Yu, Gong X. The magnetic field dependent dynamic properties of magnetorheological elastomers based on hard magnetic particles. Smart Mater Struct 2017;26:075012.

7. Stepanov GV, Borin DY, Kramarenko EY, Bogdanov VV, Semerenko DA, Storozhenko PA. Magnetoactive elastomer based on magnetically hard filler: synthesis and study of viscoelastic and damping properties. Polym Sci Ser A 2014;56:603-13. 
8. Stepanov GV, Borin DY, Storozhenko PA. Rotation of magnetic particles inside the polymer matrix of magnetoactive elastomers with a hard magnetic filler. JMMM 2017;431:138-40.

9. Stepanov GV, Borin DY, Bakhtiiarov AV. Magnetic properties of hybrid elastomers with magnetically hard fillers: rotation of particles. Smart Mater Struct 2017;26:035060.

10. Stepanov GV, Borin DY, Bakhtiiarov AV, Storozhenko PA. Negative coercivity of magnetic elastomers filled with magnetically hard particles. JMMM 2019;498:166125.

11. Jolly MR, Carlson JD, Munoz BC. The magnetoviscoelastic response of elastomer composites consisting of ferrous particles embedded in a polymer matrix. J Intell Mater Syst Struct 1996;7: 613-22.

12. Jolly MR, Carlson JD, Munoz BC. A model of the behaviour of magnetorheological materials. Smart Mater. Structure 1996;5:607-14.

13. Zrínyi M, Barsi L, Büki A. Deformation of ferrogels induced by nonuniform magnetic fields. J Chem Phys 1996;104:8750-4.

14. Barsi L, Büki A, Szabó D, Zrinyi M. Gels with magnetic properties. Prog Colloid Polym Sci 1996;102: 57-63.

15. Zrínyi M, Barsi L, Szabó D, Kilian H-G. Direct observation of abrupt shape transition in ferrogels induced by nonuniform magnetic field. J Chem Phys 1997;106:5685.

16. Zrínyi M, Barsi L, Büki A. Ferrogel: a new magneto-controlled elastic medium. Polym Gels Netw 1997;5:415-27.

17. Mitsumata T, Ikeda K, Gong J, Osada Y, Szabro D, Zrinyi M. Magnetism and compressive modulus of magnetic fluid containing gels. J Appl Phys 1999;85:8452-5.

18. Mitsumata T, Abe N. Magnetic-field sensitive gels with wide modulation of dynamic modulus. Chem Lett 2009;38:922-3.

19. Mitsumata T, Kosugi Y, Ouchi Sh. Effect of particles alignment on giant reduction in dynamic modulus of hydrogels containing needle-shaped magnetic particles. Prog Colloid Polym Sci 2009; 136:163-70.

20. Ouchi Sh, Mitsumata T. Magnetorheological effect of magnetic gels containing $\mathrm{Fe}_{2} \mathrm{O}_{3}$. Trans Mater Res Soc of Japan 2009;34:459-60.

21. Lokander M, Reitberger T, Stenberg B. Oxidation of natural rubber-based magnetorheological elastomers. Polym Degrad Stabil 2004;86:467-71.

22. Lokander M, Stenberg B. Performance of isotropic magnetorheological rubber materials. Polym Test 2003;22:245-51.

23. Lokander M, Stenberg B. Improving the magnetorheological effect in isotropic magnetorheological rubber materials. Polym Test 2003;22:677-80.

24. Endo H, Kato Sh, Watanebe M, Kikuchi T, Mitsumata T. Magnetically tunable vibration transmissibility for polyurethane magnetic elastomers. Polymers 2018;10:104.

25. Boczkowska A, Awietjan SF. Smart composites of urethane elastomers with carbonyl iron. J Mater Sci 2009;44:4104-11.

26. Boczkowska A, Awietjan SF. Magneto-rheological urethane elastomers activated by a magnetic field. Polimery 2009;54:26-30.

27. Boczkowska A, Awietjan SF. Urethane magnetorheological elastomers - manufacturing, microstructure and properties. Solid State Phenom 2009;154:107-12.

28. Kantorovich SS, Ivanov AO, Rovigatti L, Tavares JM, Sciortino F. Nonmonotonic magnetic susceptibility of dipolar hard-spheres at low temperature and density. Phys Rev Lett 2013;110: 148306.

29. Prokopieva TA, Danilov VA, Kantorovich SS, Holm C. Ground state structures in ferrofluid monolayers. Phys Rev E 2009;80:031404.

30. Borin DY, Odenbach S, Zubarev A. Non-ergodic tube structures in magnetic gels and suspensions. Soft Matter 2018;14:8537-44. 
31. https://magnetolab.ru/osn_napr_elast_en.html.

32. Bakhtiiarov AV, Stepanov GV, Storozhenko PA. Patent RU 2684295, 2018.

33. Sorokin VV, Ecker E, Stepanov GV, Shamonin M, Monkman GJ, Kramarenko EY, et al. Experimental study of magnetic field enhanced Payne effect in magnetorheological elastomers. Soft Matter 2014;10:8765-76.

34. Stepanov GV, Abramchuk SS, Grishin DA, Nikitin LV, Kramarenko EY, Khokhlov AR. Effect of a homogeneous magnetic field on the viscoelastic behavior of magnetic elastomers. Polymer 2007; 48:488-95. 